Review

\title{
Role of Endometrial Autophagy in Physiological and Pathophysiological Processes
}

\author{
Shaoliang Yang ${ }^{1}$, Haiyan Wang ${ }^{2}$, Dajin $\mathrm{Li}^{1}$, Mingqing $\mathrm{Li}^{1,3}$ \\ 1. NHC Key Lab of Reproduction Regulation (Shanghai Institute of Planned Parenthood Research), Hospital of Obstetrics and Gynecology, Fudan University, \\ 200080, People's Republic of China \\ 2. Department of Gynecology of Integrated Traditional Chinese and Western Medicine, Hospital of Obstetrics and Gynecology, Fudan University, Shanghai, \\ 200011, People's Republic of China \\ 3. Shanghai Key Laboratory of Female Reproductive Endocrine Related Diseases, Shanghai 200011, People's Republic of China
}

$\square$ Corresponding author: Email: mqli@fudan.edu.cn; NHC Key Lab of Reproduction Regulation (Shanghai Institute of Planned Parenthood Research), Hospital of Obstetrics and Gynecology, Fudan University, Shanghai, China; Tel: 86-21-33189900-2002; Fax: 86-21-33189900.

(c) Ivyspring International Publisher. This is an open access article distributed under the terms of the Creative Commons Attribution (CC BY-NC) license (https://creativecommons.org/licenses/by-nc/4.0/). See http://ivyspring.com/terms for full terms and conditions.

Received: 2018.11.23; Accepted: 2019.04.24; Published: 2019.06.09

\begin{abstract}
Endometrium is the mucosal lining of the uterus which expressed a cyclic process of proliferation, secretion and scaling under the control of hormones secreted by the ovary, and it also plays an indispensable role in the embryo implantation, the constitution of fetal-maternal interface, and the maintaining of pregnancy. In pathophysiological conditions, the abnormality or disorder of endometrium may lead to endometrium-related diseases, such as endometriosis, endometrium hyperplasia and even endometrial carcinoma. In recent years, more and more evidence revealed that autophagy exists in both the endometrium stroma cells and epithelial cells, and the activity of autophagy is changed in the different phases of menstruation, as well as in the endometrium-related diseases. Here, we aim to review the activity level, the regulatory factors and the function of autophagy in physiological and pathophysiological endometria, and to discuss the potential value of autophagy as a target for therapies of endometrium-related diseases.
\end{abstract}

Key words: autophagy, endometrium, endometriosis, endometrial carcinoma

\section{Introduction}

Autophagy is a highly conserved biological behavior in eukaryotic cells to disassemble unnecessary or dysfunctional components of the cell under exquisite regulation mechanism which can be initiated under the conditions of hypoxia, starvation, lack of nutrition, or extreme $\mathrm{pH}$ values [1-3]. In macroautophagy (commonly referred to autophagy), substances such as proteins, organelles or cytoplasm are encapsulated in a double-membraned vesicle named as autophagosome, and degraded into small molecules for reuse by the fusion of autophagosome with lysosome [4]. Autophagy can also dispose the misfolded proteins, aging organelles, and other damaged cell components to maintain the homeostasis of the cells. Basic level of autophagy is essential for almost all of the eukaryotic cells and many cellular activities, and the absence or aberration of autophagy may lead to a series of pathological states.
Recent studies revealed that autophagy also plays an indispensable role in the physiological and pathophysiological processes related to the endometria, including the cyclic menstruation, the decidualization or reconstitution during normal pregnancy, and the endometrium-related diseases such as endometriosis, endometrial carcinoma and infertility. As a basic biological activity, autophagy is closely related to the cell proliferation and apoptosis, which ubiquitously occur in the dynamic refreshment of endometrium. What's more, the autophagy level in endometrium cells reflects the condition of the disease or physiological process, and can influence the function of cells in some way. Therefore, autophagy in endometrium needs to be further studied and may be beneficial for the future treatment of endometriumrelated diseases. And this article is to review the present studies about this topic. 


\section{The level of autophagy in endometrium}

Endometrium is the mucosal lining of the uterus which can be derived into two functional layers: the transient superficial stratum functionalis and the permanent deeper stratum basalis [5]. The superficial stratum functionalis, which is completely shed and regenerated in menstrual cycle, mainly contains glandular epithelium and stromal cells, while the stratum basalis is postulated to be the place in which the stem cells reside [6, 7]. In addition, in both layers there exist the blood vessels and immune cells, whose number varies in different phase of a menace cycle in normal endometrium, including NK cells, macrophages, DC cells and so on[8, 9]. Autophagy is regarded as a ubiquitous physiological process that occurs in all eukaryotic cells. There is no doubt that autophagy also exists in all of the endometrial cells and plays diverse function in different process and diseases, but it was only reported to be detected in endometrial stroma cells and epithelial cells; study is deficient in illustrating the autophagy level in endometrial immune cells and vascular endothelial cells. This may be related to the difficulty in methods to monitoring the autophagy level and the acquiring enough cells for the experiment.

As a marker of autophagy, LC3 was found to be predominantly expressed in epithelial relative to stromal cells in normal endometrium [10] and eutopic or ectopic endometria form endometriosis patients [11]. Autophagy in glandular cells was very weakly positive in the early and late proliferative phases; In contrast, intense immunoreactivity for LC3 was detected during the secretory phase and peaked during the late secretory phase of the menstrual cycle in glandular cells [10, 12]. A withdraw of progesterone or estrogen can significantly increase the expression level of LC3 in endometrial Ishikawa cells, which may imitate the changes in menstrual phase [10]. In a murine ovariectomized rat model, autophagosomes was observed to be significantly increased in the cytoplasm of the uterine epithelial cells 4 weeks postoperation compared with the shamoperated rats; similarly, the level of autophagy was higher in postmenopausal uterine epithelial cells compared to premenopausal uterine epithelial cells [13]. Autophagy was also reported to exhibit a dynamic pattern during pregnancy. In the mouse model, LC3 was observed to reach the highest level during days 1 and 2 of pregnancy, and gradually fall from day 4 to day 8 . The LC3 level on day 1 of pregnancy can be effectively inhibited by dexamethasone, which is a widely used anti-inflammatory compound, indicating that inflammatory response may be associated with the increased autophagy in the day 1 pregnant uterus [14].
Similar to endometrial epithelium, the level of autophagy in normal endometrial stroma cells (ESCs) significantly increased in secretory phase compared with proliferative phase; while in ectopic ESCs from endometriosis patients, autophagy maintained a constant level throughout the menstrual cycle $[12,15]$. And during the pregnancy, the autophagy of ESCs was also in a dynamic change, but there was no consecutive observation in human decidua $[14,16]$. Autophagy in ESCs is relatively low compared to glandular cells; LC3 expression in normal ESCs is quite weak even in the secretory phase [10]. What draw our interest is that autophagy level differs in normal ESCs, eutopic ESCs and ectopic ESCs. Actually, there exist contrary opinions on whether autophagy is stronger in normal tissue or endometriotic tissues. Some researchers proved that autophagy level were significantly reduced in eutopic ESCs, ectopic ESCs, serum and peritoneal fluid from women with endometriosis compared with normal women $[12,15,17-20]$, while others held the opposite views, revealing that autophagy showed a significant up-regulation in ectopic endometrium compared with normal endometrium [21-24]. Similarly, the comparison of autophagy in ectopic ESCs and eutopic ESCs was also not completely clear and consensus [11, 15, 21-24]. The differences in researches may due to the different detective method, the individual characteristic of specimen, and the immune microenvironment of patients. Thus, further studied are still needed to find out the truth.

Human endometrium undergoes repetitive cyclic regeneration after the shedding off in menstruation. In the dynamic change, endometrium-derived human mesenchymal stem cells (hMESCs) resided in the basal layer have contributed a lot to enable the rapid regeneration of endometrium after the shedding off in last cycle. The basic level of autophagy seems to be low in hMESCs, while under oxidative stress, the suppression of the p53 transcriptional activity or the calcium chelation caused a significantly increase of autophagy and lead to cell death under oxidative stress $[25,26]$. Autophagy was discovered to induce G0/G1 arrest and apoptosis of menstrual blood-derived endometrial stem cells via GSK3 $\beta / \beta$-catenin pathway [27]. On the other hand, in endometrial cancer stem cells (ECSCs), autophagy level seems to be relatively strong. CD133 and CD44 are reported to be important markers of ECSCs. The results from Ran et al. showed that Beclin1, Atg5, Atg7 mRNA levels were significantly enhanced but the P62 was obviously reduced in $\mathrm{CD} 133^{+} / \mathrm{CD} 44^{+}$ cells compared to normal JEC cells (a cell line of endometrial cancer), demonstrated that the $\mathrm{CD}_{133^{+}}$/ $\mathrm{CD}_{4}{ }^{+}$cells which exhibit CSC properties possess 
higher autophagy [28] (Table 1).

Table 1. The autophagy level in endometrium

\begin{tabular}{|c|c|c|c|c|c|c|}
\hline & \multicolumn{3}{|l|}{ Non pregnant } & \multicolumn{3}{|l|}{ Pregnant } \\
\hline & Proliferative phase & Secretory phase & Menstrual phase & First trimester & Second trimester & Term \\
\hline Stromal cells & $+(-)[10,15]$ & $++[10,15]$ & / & $+++[14]$ & $+[14]$ & $++[16]$ \\
\hline Epithelial cells & $+[10]$ & $+++[10]$ & $++[13]$ & $+[14]$ & / & / \\
\hline endometrium-derived human mesenchymal stem cells & \multicolumn{6}{|c|}{ Induced by calcium chelation or p53 suppression under oxidative stress $[25,26]$} \\
\hline
\end{tabular}

+++: The autophagy level in normal endometrial epithelial cells in the secretory phase; -: Negative expression of autophagy; /: Not mentioned

\section{Regulatory mechanism of autophagy in endometrium}

Autophagy takes part in variety of biological activities, and the level of autophagy can influence the cellular metabolism, the proliferation and program cell death, and the drug resistance or sensibility of cancer cells. Thus, autophagy is precisely regulated in different condition. Factors such as hormones, cytokines, metabolic products and non-coding RNAs can regulate the autophagy level independently or interdependently.

\section{Hormones}

Hormones are the main factors that regulate the cyclic proliferation, secretion and shedding of endometrium. Considering that autophagy also changes during the menstrual cycle, it's not difficult to imagine that hormones play important roles in adjusting the level of autophagy in endometrium.

Estrogen is the predominant hormone during the proliferative phase, and many researches have proved that it can inhibit the autophagy activity in endometrium $[13,15,17,29,30]$. Mei et al. found that estradiol $\left(\mathrm{E}_{2}\right)$ treatment induced a marked increase in CXCL12 secretion and CXCR4 expression together with decreases in autophagy grade, punctuate LC3B immunofluorescent staining and autophagyassociated protein levels, while the suppression of autophagy induced by E2 and recombinant human CXCL12 protein could be abrogated by an anti-CXCR4 neutralizing antibody, suggesting that estrogen could suppress the autophagy of ESCs by upregulating CXCL12 and CXCR4 expression [29]. Estrogen was also reported to induce the autophagy level in normal endometrial cancer cells and endometrial carcinoma stem cells by promoting the expression of estrogen induced gene 121(EIG121), which can induce cytoplasmic vacuolization, induce the formation of autophagosomes and enhance lysosomal degradation of long-lived proteins [31]. Knockdown of EIG121 compromises starvationinduced autophagy and sensitizes cells to cell death induced by nutrient deprivation and exposure to cytotoxic agents [28, 31]. In a ovariectomized rats menopausal model, autophagy was observed to be increased in uterine endometrium when estrogen was deprived, and the estrogen depletion induced autophagy was possibly via endoplasmic reticulum (ER) stress-dependent arachidonic acid / prostaglandin E2 axis inhibition and Akt-mTOR signaling pathway suppressing in uterine epithelial cells [13].

Relationship between estrogen and progestogen can be both synergistic and antagonistic depending on the tissues or cells receiving the signals. As is the case, their role in regulating the activity of autophagy may be complicated. In the study of Mei et al., the estrogen induced autophagy in ESCs could be reversed by progestogen [15], suggesting that progestogen plays an antagonistic role in controlling the autophagy in ESCs. Consistent with this, Choi et al. [32] found that dienogest, but not progestogen, can enhance autophagy induction in endometriotic cells by impairing activation of AKT, ERK1/2, and mTOR signal pathways. On the contrary, Choi et al. [14] found that progesterone and estrogen both reduced the autophagic response in the uteri in ovariectomized mice model. The differences may occur from the tested samples, actually, the mice uteri contain different kinds of cells, and the total mRNA or protein may not reflect the separate change of autophagy in ESCs, EECs, or other cells precisely.

Other hormones are also involved in regulating the autophagy condition in endometrium. After menopause, the level of estrogen and progestogen goes down while follicle-stimulating hormone (FSH) commonly rises with the decline of ovary function. It was reported that high circulating levels of FSH in postmenopausal women activated the phosphorylation of Smad2/ Smad3 through transforming growth factor beta receptor II (TbRII). The complexes of phosphorylated Smad2/Smad3 subsequently transported into the nucleus and launched the expression and activation of several autophagy-related molecules such as ATG5, ATG12, ATG3, and ATG7. Persistent activation of cell autophagy may directly or indirectly induce cell apoptosis. Thus, FSH can promote the autophagy of endometrial adenocytes [33]. Autophagy may also be related with increased androgen availability in PCOS. It was found that autophagy related genes were significantly reduced in anovulatory PCOS compared to healthy endometrium, and was negatively correlated with free androgen index. Treatment of PCOS patients with metformin 
significantly increased the endometrial mRNA levels of autophagy-associated genes, suggesting that the increased androgen level in PCOS is associated with metformin-sensitive transcriptional downregulation of endometrial autophagy [34]. And dexamethasone, a widely used anti-inflammatory compound, was also observed to inhibit the autophagy of mice uteri at the first day of pregnancy, when the autophagy level should have been the highest [14]. This effect suggests that inflammatory response may be associated with increased autophagy in the day 1 pregnant uterus. Other hormones, such as luteinizing hormone (LH), anti mullerian hormone (AMH), prolactin (PRL), and Human Chorionic Gonadotropin (HCG), which also take vital part in the menstruation cycle or gestation of fertile women, still need to be investigated on their role of regulating the autophagy of endometrium.

\section{Metabolism and metabolites}

Autophagy can be influenced by many metabolites, and it can affect the metabolic condition of cells simultaneously. Rhee et al. found that a high level of palmitic acid, which is commonly observed in obese people as a result of imbalance of energy metabolism, can impaired the autophagy of human endometrial cells during decidualization [16]. This may be one of the mechanisms that lead to the poor reproductive outcome and early pregnancy loss in obese women. Folate acid, a widely applied supplement by women during pregnancy, was reported to induce the autophagy of endometrium, thus benefits the decidualization process [35]. In another experiment, researchers found that compared to the control group, caloric restriction can significantly increase the autophagy activity and inhibit the p-Akt and mTOR levels in endometriotic lesions of endometriosis mice model [36].

Apart from this, the amino acid metabolism can also regulate the autophagy condition in endometrium. It was detected that the level of phenylalanine, indoleacrylic acid (IAA), phosphocholine and lysoplatelet-activating factor-16 (lyso-PAF) in serum were higher in endometrial carcinoma patients than normal controls, and all of them played a role in regulating the autophagy tumor cells. PAF can induce tumor cell apoptosis and autophagy, while IAA triggered apoptosis and had a biphasic effect on autophagy: inhibiting autophagy with doses $<1 \mathrm{mmol} / \mathrm{L}$ but inducing at $1 \mathrm{mmol} / \mathrm{L}$. Interestingly, the alterations in apoptosis and autophagy caused by $1 \mathrm{mmol} / \mathrm{L}$ IAA coule be reversed by the concomitant treatment of tryptophan (100 mumol/L). Phosphocholine inhibited tumor cell invasion and migration, and promoted cell proliferation and autophagy, all in a dose-dependent manner [37]. Other substance, such as retinoic acid, was also reported to enhance the autophagy in ESCs [38]. According to this, we can learn that autophagy is widely regulated by many kinds of metabolic products, and closely related to various biological activities.

What's more, the supply of oxygen plays a critical role in the process of cell growth. The lack of oxygen, namely hypoxia, can induce the autophagy of cultured human endometrial stromal cells (HESCs) in a time-dependent manner through hypoxia-inducible factor-1alpha (HIF-1a) [23]. LC3-II expression and the number of autophagosomes were gradually increased by hypoxia treatment [22]. This autophagy inducing effect of hypoxia was also reported in other cells and diseases, such as preeclampsia, non-alcoholic steatohepatitis, neurodegenerative diseases, and so on [39, 40]. Oxidative stress is another good candidate responsible for autophagy induction in endometriotic cells. The persistent oxidative stress, by itself and/or a positive feedback with heme oxygenase-1(HO-1), reactive oxygen species (ROS), and other downstream response, can stimulate the autophagic process [41-43].

Oxidative stress induces a rapid calcium release from intracellular stores, which play an important role in apoptosis and necrosis, senescence, as well as autophagy. With a sublethal $\mathrm{H}_{2} \mathrm{O}_{2}$ treatment, an artificial environment of oxidative stress was formed. It was found that in $\mathrm{H}_{2} \mathrm{O}_{2}$-treated human endometrium-derived stem cells, intracellular calcium $([\mathrm{Ca} 2+] \mathrm{i})$ chelation by BAPTA-AM can effectively initiate an early onset of autophagy, as observed by both the phosphorylation status of AMPK/mTORC1 pathway and the dynamics of the LC3 lipidization. In other word, the calcium release under oxidative stress could inhibit the activation of autophagy, which may be related to the prevention of the premature senescence and DNA damage [26]. Different from the free state of calcium, iron is presented in cells as ferric ammonium citrate (FAC). Treatment with FAC can significantly increase the protein level of LC3-II and the number of autophagosomes in a dose dependent manner in immortalized endometriotic Cells, by the activation of AKT and MAPK pathway [41].

\section{Enzymes, inhibitor and drugs}

Serum and glucocorticoid-regulated kinase 1 (SGK1) is a serine/threonine protein kinase that shares structural and functional similarities with the AKT family of kinases [44]. It plays an important role in cellular stress response by activating certain potassium, sodium, and chloride channels, suggesting an involvement in the regulation of processes such as cell survival, neuronal excitability, and renal sodium excretion [45-47]. In endometrial carcinoma, the 
expression of SGK1 was upregulated in tumor tissues than normal control, and inhibiting SGK1 with SI113 can induce a reduction of endometrial cancer cells viability as well as a significant increase of autophagy, as revealed by the increase of the markers LC3B-II and beclin I, detected by both immunofluorescence and western blot analysis [48]. This effect may be associated to the induction of endoplasmic reticulum stress markers GRP78 and CHOP evaluated by both real-time PCR and western blot analysis.

Inhibitors of the key enzymes can also influence the process of autophagy in endometrium. Liraglutide, which is an agonist of glucagon-like peptide-1 receptor (GLP-1R), can significantly induced autophagy of endometrial carcinoma cells and inhibited Ishikawa cell growth, as well as the elevated AMPK expression [49]. Bortezomib is an inhibitor of $26 \mathrm{~S}$ proteasome, which has been reported to inhibit protein degradation in lysosomesmay by inducing ERK phosphorylation and suppress cathepsin B in endometrial carcinoma cells and enhance chemotherapy efficacy by abolishing chemotherapy-related autophagy [50]. A new synthetic histone deacetylase inhibitor, MHY2256, was found to induce apoptosis and autophagy cell death in endometrial cancer cells via p53 acetylation [51]. Similarly, as an inhibitor of mTOR, RAD001 significantly decreased phosphorylation of $\mathrm{mTOR}$ and inhibited proliferation of progestinresistant cancer cells and induced a higher sensitivity to paclitaxel-induced apoptosis by promoting autophagy $[52,53]$. Rapamycin is another inhibitor of mTOR, which is the most commonly used as an inducer of autophagy, but we should notice that it can affect both mTORC1 and mTORC2. What's more, mTOR is a major regulatory protein that is part of several signaling pathways, including for example those that respond to INS/insulin, EGF/epidermal growth factor and amino acids, and it thus controls process other than autophagy [54-57]. Therefore, rapamycin will ultimately affect many metabolic pathways and the pleiotropic effects need to be considered [58].

Many drugs, especially those used in the anti-tumor treatment, were discovered with the effect of adjusting the autophagy activation. Metformin, a commonly used medicine for treating type II diabetes, which has recently been reported to possess anti-proliferative properties in a variety of cancers, was reported to regulate the autophagy of tumor cells $[59,60]$.It was found that metformin could increase the apoptosis, inhibited the growth, and promote the autophagy of endometrial carcinoma cells with different concentrations in a dose- and time-dependent manner [59-62]. And the autophagy inducing effect was proved to be related with CEBPD or AMPKa [63,
64] in several tumor cells. Sorafenib is another anti-tumor drug, or more precisely, a multi-target tyrosine and serine/threonine kinase inhibitor approved by FDA for the treatment of hepatocellular carcinoma, advanced renal carcinoma and radioactive iodine-resistant thyroid carcinoma $[65,66]$. Sorafenib could induce an unfolded protein response (UPR) in EC cells that connects with an autophagic output through a MAPK/JNK-dependent mechanism and this MAPK/JNK-dependent early protective autophagic response in endometrial carcinoma cells may count for the limited effect of sorafenib in a multi-institutional phase II trial in advanced uterine carcinoma patients [67]. Programmed cell death 4 (PDCD4), a newly identified tumor suppressor, was uncovered to effectively inhibit the proliferation and suppress the migration and invasion of endometrial cells by inhibiting cell autophagy, probably through NF-kB/MMP2/MMP9 signal pathway [68]. Cisplatin was also reported to upregulate cell autophagy in endometrial cancer cells via the PI3K/AKT/mTOR signaling pathway [69]. And interestingly, the combination of cisplatin and rapamycin showed a synergetic anti-endometrial cancer effect [58]. Also, other drugs [70], including traditional Chinese medicine such as Isoliquiritigenin (ISL), Protopanaxadiol (PPD) showed a potential to regulate the autophagy in endometrium [30,71, 72]. The role of autophagy in endometrial cancer is worthy investigating and is discussed in the next part.

\section{Others}

With the constant-depth study, non-coding RNA has been unveiled to participate in more and more biological activities. It was now clear that many micro RNA and long non-coding RNA (lncRNA) have a regulatory effect on the activation of autophagy in endometrium. For example, miR-101-3p was reported to upregulate the expression of autophagy-related proteins LC3-II and beclin-1 in EC cells in a time- and dose-dependent manner, by downregulating the expression of enhancer of zeste homolog 2 (EZH2) [73]. EZH2 is a critical component of the polycomb repressive complex 2 with intrinsic histone methyl transferase activity that mediates gene silencing by catalyzing trimethylation on lysine 27 of histone $\mathrm{H} 3[74]$. And it can epigenetically repress tuberous sclerosis 2 (TSC2), a negative regulator of the mTOR pathway. Downregulation of TSC2 by EZH2 elicited mTOR activation, which in turn induced the inhibition of autophagy [75]. MiR-210, a micro RNA which was significantly induced by hypoxic treatment, was found to promote autophagy partly by inhibiting the Bcl-2 expression [76]. MiR-218 can also inhibit the autophagy of endometrial cancer cells by 
directly bind to the 3'-UTR of HMGB1 gene, which is one of the most important regulators of cellular autophagy [77]. lncRNAs, specifically HOTAIR, can regulate the level of autophagy in human endometrial cancer cells by influencing Beclin-1, MDR, and P-gp expression. Interference of HOTAIR enhanced the autophagy activity of cisplatin-resistant Ishikawa cells and further reduced the proliferation of cisplatinresistant Ishikawa cells [78]. IncRNA-MALAT1 also promoted hypoxia-induced autophagy in endometrial stromal cells and reduced their apoptosis, which facilitated the survival of ectopic endometrium[79].

DNA mismatch repair (MMR) can induce autophagy in human endometrial tumor cells via signal transferred by p53 after exposure to 6-thioguanine (6-TG), a chemotherapy drug which can be recognized by MMR. Autophagy induced by DNA mismatch repair inhibits apoptosis of endometrial carcinoma cells. An inhibition of autophagy by Atg5 knockdown will significantly promotes the apoptosis of cancer cells after DNA mismatch repair processing of 6-TG, suggesting that autophagy mediated the survival of cancer cells with MMR in the treatment of 6-TG [80]. A brief summary of regulators of autophagy in endometrium is shown in Table 2.

\section{The role of autophagy in physiological conditions}

As is mentioned above, the level of autophagy is in a dynamic state during the menstrual cycle. The role of autophagy in menstruation is still not quite clear. Based on the limited research, we can learn that autophagy may be related to the apoptosis induction of endometrial cells by increasing the BAX: BCL2 ratio and activating the subsequent caspases, and is directly involved in the cyclic remodeling of the human endometrium [10].

Table 2. Regulatory factors of autophagy in endometrium

\begin{tabular}{|c|c|c|c|c|}
\hline & & $\begin{array}{l}\text { Effect on } \\
\text { autophagy }\end{array}$ & Note & References \\
\hline \multirow[t]{7}{*}{ Hormones } & \multirow[t]{2}{*}{ Estrogen } & downregulate & by upregulating CXCL12 and CXCR4 expression & {$[13-15,17,29,30]$} \\
\hline & & upregulate & by promoting the expression of EIG121 & {$[28,31]$} \\
\hline & \multirow[t]{2}{*}{ Progestogen } & upregulate & impairing activation of AKT, ERK1/2, and mTOR & {$[28,31]$} \\
\hline & & downregulate & PR dependent & {$[14]$} \\
\hline & FSH & upregulate & $\begin{array}{l}\text { partly through activating the phosphorylation of Smad2/ Smad3 via transforming } \\
\text { growth factor beta (TGF } \beta \text { ) }\end{array}$ & {$[33]$} \\
\hline & Androgen & $\begin{array}{l}\text { negatively } \\
\text { correlated }\end{array}$ & $\begin{array}{l}\text { increased androgen availability in PCOS is associated with downregulation of } \\
\text { endometrial autophagy }\end{array}$ & {$[34]$} \\
\hline & Dexamethasone & downregulate & $\begin{array}{l}\text { significantly reduced autophagy in the day } 1 \text { pregnant mice uterus which may be } \\
\text { related to inflammatory response }\end{array}$ & [14] \\
\hline \multirow{10}{*}{$\begin{array}{l}\text { Metabolism } \\
\text { and } \\
\text { Metabolites }\end{array}$} & Palmitic acid & downregulate & $\begin{array}{l}\text { defects in autophagy caused by palmitic acid may contribute to impaired } \\
\text { decidualization }\end{array}$ & [16] \\
\hline & Indoleacrylic acid (IAA) & biphasic effect & $\begin{array}{l}\text { inhibiting autophagy with doses }<1 \mathrm{mmol} / \mathrm{L} \text { but inducing at } 1 \mathrm{mmol} / \mathrm{L} \text {; } \\
\text { autophagy caused by } 1 \mathrm{mmol} / \mathrm{L} \text { IAA coule be reversed by the concomitant } \\
\text { treatment of tryptophan }(100 \mathrm{mumol} / \mathrm{L})\end{array}$ & [37] \\
\hline & Phosphocholine & upregulate & in a dose-dependent manner & [37] \\
\hline & $\begin{array}{l}\text { lyso-platelet-activating } \\
\text { factor-16(lyso-PAF) }\end{array}$ & upregulate & in a dose-dependent manner & [37] \\
\hline & retinoic acid & upregulate & / & [38] \\
\hline & Folate acid & & / & {$[35]$} \\
\hline & calcium & downregulate & $\begin{array}{l}\text { calcium chelator induce an early onset of autophagy through AMPK/mTORC1 } \\
\text { pathway under oxidative stress }\end{array}$ & [26] \\
\hline & iron & upregulate & by the activation of AKT and MAPK pathway & [41] \\
\hline & hypoxia & upregulate & through HIF-1a & {$[22,23]$} \\
\hline & Oxidative stress & upregulate & $\begin{array}{l}\text { by itself and/or a positive feedback with heme oxygenase-1(HO-1), ROS, and other } \\
\text { down-stream responses }\end{array}$ & [21, 41-43] \\
\hline \multirow{13}{*}{$\begin{array}{l}\text { Enzymes, } \\
\text { inhibitor and } \\
\text { drugs }\end{array}$} & SGK1 & upregulate & associated to the induction of endoplasmic reticulum stress & [48] \\
\hline & Liraglutide & upregulate & may be related to the AMPK phosphorylation & [49] \\
\hline & Bortezomib & downregulate & inducing ERK phosphorylation and inhibiting protein degradation in lysosomes & [50] \\
\hline & MHY2256 & upregulate & p53 acetylation & [51] \\
\hline & RAD001 & upregulate & downregulation of $\mathrm{AKT} / \mathrm{mTOR}$ phosphorylation & {$[52,53]$} \\
\hline & Rapamycin & upregulate & binds to FKBP1A/FKBP12 and inhibits mTORC1 & {$[30,58]$} \\
\hline & Metformin & upregulate & inducing autophagy through CEBPD upregulation or AMPKa & {$[63,64]$} \\
\hline & Sorafenib & upregulate & through a MAPK/JNK-dependent mechanism & [67] \\
\hline & Isoliquiritigenin (ISL) & upregulate & By activating the extracellular signal regulated kinase signaling pathway & [71] \\
\hline & Itraconazole & upregulate & inhibit the expression of mTOR signaling components & [70] \\
\hline & Protopanaxadiol & upregulate & via AMPK/JNK phosphorylation & {$[30,72]$} \\
\hline & PDCD4 & downregulate & may be related to NF-kappaB/MMP2/MMP9 signal pathway & {$[68]$} \\
\hline & Cisplatin & upregulate & via the PI3K/AKT/mTOR signalling pathway & [69] \\
\hline \multirow[t]{6}{*}{ Others } & miR-101-3p & upregulate & by downregulating the expression of Enhancer of zeste homolog 2 (EZH2) & [73] \\
\hline & $\operatorname{miR}-210$ & upregulate & partly by inhibiting the Bcl-2 expression & [76] \\
\hline & MiR-218 & downregulate & by directly bind to the 3 -UTR of HMGB1 gene & [77] \\
\hline & Lnc-RNA HOTAIR & downregulate & by influencing Beclin-1, MDR, and P-gp expression & [78] \\
\hline & lncRNA-MALAT1 & upregulate & / & [79] \\
\hline & DNA mismatch repair (MMR) & upregulate & via signal transferred by $\mathrm{p} 53$ & [80] \\
\hline
\end{tabular}


In the mechanism research of keratinocyte growth factor in treating intrauterine adhesion, autophagy was confirmed to show a close relatively relationship with the proliferation of endometrial epithelial cells and the angiogenesis in endometrial [81]. This may also occur in the menstrual period after the shedding of endometrium. Accordingly, autophagy in endometrial cells may be related to the remodeling of the endometrium, as well as the angiogenesis during the menstruation cycle.

After menopause, endometrium undergoes a physiological atrophy with the withdrawal of estrogen and progestogen. Autophagy is detected to play a prominent role in uterine epithelial cell death and is a critical regulator of the uterine epithelium that accounts for endometrial atrophy [13]. Similarly, persistent activation of cell autophagy induced by the high level of FSH may directly or indirectly induce cell apoptosis, and finally contribute to the atrophy of endometrial [33]. What's more, 3-MA injections significantly increases uterine glycogen content in ovariectomized uteri, suggesting that heightened autophagy under hormone deprivation is associated with glycogen breakdown as an energy provider [14].

Another role of autophagy is the regulatory effect during decidualization. Autophagy pathway plays a vital role in cell proliferation, endometrial function, prostaglandin secretion and cell attachment [82]. A high level of autophagy was detected at the first day of pregnancy in mice uteri when the uterus shows an inflammatory response to mating, revealing that uterine autophagy may have undetermined functions as a responsive mechanism to acute inflammation [14]. During the decidualization period, the autophagy in endometrium significantly increased, and an impaired autophagy in obese women may be related to a poor outcome of pregnancy, suggesting that autophagy contribute to the endometrial receptivity and embryo implantation [16]. Being related to endoplasmic reticulum stress, autophagy was also reported to contribute to early pregnancy success and regulate endometrial function [83]. In conclusion, the increasing autophagy is indispensable during the decidualization of endometrium and defects in autophagy may relate to poor reproductive outcomes and early pregnancy loss.

\section{The role of autophagy in endometrium-related diseases}

As a fundamental activity of cells, autophagy is essential in biological process of endometrium in both physiology and pathology. In endometrium-related diseases, autophagy plays a multifunctional and complicated role as revealed by more and more evidence, which increased the difficulties for us to understand the effect of it in a certain situation. As a matter of fact, the function of autophagy in different endometrium-related diseased may be totally different. Here, we make a brief discussion of autophagy in endometrium-related diseases separately based on the researches till now, and more thorough studies are needed to help us understand the role of autophagy in endometrium.

\section{Endometrial carcinoma}

Endometrial carcinoma is one of the most common malignant diseases in women all over the world. It was reported that autophagy has played important roles in the tumorigenesis, the proliferation, the drug resistance, and the immune regulation of endometrial carcinoma. To investigate the autophagy targeted treatment for endometrial carcinoma may be a potential way instead of the traditional method.

Three autophagy related genes, RB1CC1, ULK4, and WDR45 were found to be significantly mutated in endometrial carcinoma patients compared with normal women, suggesting that aberrant activation or disruption of autophagy plays a role in the tumorigenesis of endometrial carcinoma [84]. LC3A reactivity was recognized in three basic patterns: diffuse cytoplasmic, cytoplasmic/juxta-nuclear, and the so-called "stone-like" structures (SLS). The latter has emerged as the hallmark of autophagic activity, being detected exclusively in endometrial carcinomas and mildly in the atypical hyperplasias, while other forms of hyperplasia without cytological atypia and normal endometrial tissues expressed only cytoplasmic staining patterns, suggesting that autophagy is more activated in endometrial carcinoma cells than normal or hyperplastic endometrial [85]. The highly activated autophagy may be related the proliferation, apoptosis and other biological activity of tumor cells, which contribute to the oncogenesis of endometrial carcinoma.

Actually, it was reported that autophagy may promote cell survival under nutrient deprivation and other cellular stresses [31]. But in other cases, autophagy is more likely to associate with the cell apoptosis and cell death, which is negatively related to proliferation. Several anti-cancer drugs was found to induce the autophagy of cancer cells and inhibit the cell viability, suppress the cell proliferation and downregulate the cell growth $[48,49,86]$. Similarly, the lower level of autophagy in progestogen-resistant endometrial cancer cells was related to the increased proliferation [52], and estrogen was also found to promote the cell proliferation and simultaneously inhibit the autophagy in endometrial cancer cells [30]. In conclusion, autophagy may play a beneficial role in 
the initiation of endometrial carcinoma and the survival of tumor cells under stress, but excessive autophagy may lead to the apoptosis and cell death, which is bad for the proliferation of endometrial carcinoma.

Autophagy is also related to the drug resistance of endometrial carcinoma cells for some traditional anti-tumor medicines. For example, cisplatin was reported to upregulate cell autophagy in endometrial cancer cells via the PI3K/AKT/mTOR signalling pathway [69]. And by inhibiting the level of autophagy, chloroquine can effectively suppress the proliferation in endometrial cancer cell lines in a dose-dependent manner, the sensitivity of endometrial carcinoma cells to cisplatin was also improved by knocking down ATG5 or ATG7 [87]. Autophagy has also been shown to be involved in cell resistance to paclitaxel. Paclitaxel-mediated cell death was further potentiated by pretreatment with autophagy inhibitor chloroquine or shRNA against the autophagic gene beclin 1, suggesting that paclitaxelelicited autophagic response plays a protective role that impedes the eventual death of endometrial carcinoma cell, and autophagy-inhibitor therapy could be an effective and potent strategy to improve paclitaxel treatment outcomes in the treatment of endometrial carcinoma [88]. This kind of protective auphagic response was also observed in some new drugs, such as sorafenib. Sorafenib is currently used to treat hepatocellular carcinoma, advanced renal carcinoma and radioactive iodine-resistant thyroid carcinoma, but it has not showed much effect in a multi-institutional phase II trial in advanced uterine carcinoma patients. A recent study shows that targeting autophagy enhances sorafenib cytotoxicity and suppresses tumor growth and pulmonary metastasis progression, which can provide insights into the unopposed resistance of advanced endometrial carcinoma to sorafenib and highlight a new strategy for therapeutic intervention in recurrent endometrial carcinoma [67]. For those patients who are insensitive to chemotherapy, inhibiting the autophagy of tumor cells may be one of the new strategies for effective treatment.

On the other hand, autophagy is necessary for the anti-tumor effect in some of the drugs, meaning that they can induce the cancer cell death at least partly by promoting the autophagy. More and more evidence showed that metformin has effects on suppressing the growth of different kinds of tumor cells. It was revealed that metformin can induce the autophagy of endometrial cancer cells, and inhibition of autophagy either by beclin1 knockdown or by 3-methyladenine-mediated inhibition of caspase-3/7 can significantly suppress the anti-proliferative effects of metformin on endometrial cancer cells, indicating that the anti-proliferative effects and apoptosisinducing effect caused by metformin were partially or completely dependent on autophagy [62]. Autophagy was also involved in the cell death inducing effect of suberoylanilide hydroxamic acid (SAHA), a histone deacetylase inhibitor, which was a promising therapeutic agent for endometrial stromal sarcoma [89]. These results told us that autophagy may be a double-edged sword in the treatment of endometrial carcinoma. Just like the relationship of autophagy and proliferation, in a certain condition, autophagy may promote the drug resistance or the drug effect according to specific circumstances.

Apart from this, autophagy is related to the immune regulation of the local tumor environment. Exposure with rapamycin results in a significantly increased autophagy in endometrial cancer cells, and it can further enhanced the cytotoxicity of NK cells by upregulating the expression of IL-27 in endometrial cancer cells and IL-27 receptors on NK cells, which can restricted the growth of tumor in Ishikawaxenografted nude mice [58]. In contrast, high expression of Beclin-1 is related to high tumor grade, high myometrial invasion, and a poor 5-year survival in endometrioid adenocarcinoma $[90,91]$.

\section{Endometriosis}

Endometriosis is a chronic, estrogen-dependent disease and characterized by the implantation of endometrial glands and stroma deep and haphazardly into the outside the uterine cavity [92]. Autophagy in endometriosis-derived tissues is reduced compared with normal endometriosis tissues, especially in ectopic endometrium and during the secretory phase [93]. The impaired autophagy plays critical roles in the process of cell apoptosis and proliferation, invasion and migration, cell immunity, and is associated to the stage and prognosis of endometriosis $[93,94]$.

As a benign disease characterized by the presence, transfer, invasion, and cultivation of growing endometrial tissue, endometriosis is now found to be more and more closely related to the immune microenvironment at local peritoneal cavity. The impaired autophagy in ectopic ESCs results in the downregulation of hematopoietic cellular kinase (HCK) by inactivating signal transducer and activator of transcription 3 (STAT3), as well as the increased secretion of the downstream molecules CXCL8/IL-8 and IL23-A by ESCs, and this increase induced the upregulation of CD16- NK cells and decline of cytotoxic activity in ectopic lesion microenvironment [17]. Similarly, we also found that rapamycin decrease the level of IL-15 receptors in ESCs, which may 
downregulate the effect of IL-15 on stimulating the growth and invasion of ESCs and helping the immune escape of ESCs by suppressing the cytotoxic activity of NK cells in the ectopic milieu [95]. What's more, we proved that autophagy in ectopic ESCs enhances the cytotoxic activity of NK cells and suppresses the growth of ectopic lesions in a mouse EMS model. By increasing the autophagy of ectopic ESCs, protopanaxadiol significantly increased the expression of activating natural cytotoxicity receptors (NKp30 and NKp46) and cytokine IFN- $\gamma$, and decreased IL-10 expression in NK cells, and protopanaxadiol can also decreased the number and weight of mouse ectopic lesions [29]. Autophagy in ESCs can also influence the function of macrophage around the ectopic lesion. Ruiz et al. noted that autophagic flux inhibitor hydroxychloroquine (HCQ) increase the levels of peritoneal macrophages and the IP-10 (10 kDa interferon- $\gamma$-induced protein) chemokine in a mouse model of endometriosis [11], whereas the role of macrophage in the development of endometriosis needs to be deep investigated.

Autophagy is also reported to be related to the proliferation and apoptosis of eutopic or ectopic endometrial tissues from endometriosis patients. By inhibiting the activity of autophagy in endometriotic stromal cells, MK2206 (an AKT inhibitor) and chloroquine markedly reduced cell growth and regrowth after discontinuation of treatment, and reduced the size of endometriotic implants, indicating that autophagy played a cytoprotective role in endometriosis [96]. Autophagy was also reported to inhibit the proliferation and colony formation, and the growth of filopodia of the endometriotic cell line CRL-7566 through fascin-1[20]. A newly identified tumor suppressor, PDCD4, can effectively inhibit the proliferation and colony-forming ability and suppress the migration and invasion ability of endometrial cells probably by inhibiting cell autophagy [68]. Xu et al. also showed that autophagy in ovarian endometriotic cell line CRL-7566 may contribute to pathological development of endometriosis through enhancing cell survival [76]. However, some researcher deemed that autophagy may promote the apoptosis of endometriotic cells. Dienogest treatment of endometriotic cells was found to suppress AKT and ERK1/2 activity, thereby in turn inhibiting mTOR, inducing autophagy and promoting apoptosis [32]. Choi et al. also identified that rapamycin treatment induced autophagy and led to apoptosis promotion, which was reversed by the addition of 3-MA, suggesting that mTOR inhibition promotes endometriotic cell apoptosis via autophagy induction [12]. And rapamycin was observed to inhibit the proliferation and colony formation and inhibit the growth of filopodia of the endometriotic cell line CRL-7566, which can be reversed by 3-MA [20]. Collectively, autophagy may be essential for the proliferation and cell growth of endometriotic cells, but under some certain circumstances, the increased activity of autophagy could lead to their apoptosis.

Migration and invasion are two typical characteristics of endometriotic cells contributing to the development of endometriosis, which are also uncovered to be regulated by the level of autophagy. Human endometrial epithelial cells treated by hypoxia displayed a higher level of autophagy and higher ability of migration and invasion compared to the control cells; however, when co-treated with autophagy inhibitors 3-MA and CQ, the migratory and invasive potential were dramatically decreased. These observations indicated that autophagy facilitates the hypoxia triggered migration and invasion [22]. Similar effects were also observed by inhibiting autophagy with specific inhibitors and Beclin1 siRNA in human endometrial stromal cells, the decreased autophagy activity attenuated their hypoxia triggered migration and invasion [23]. PDCD4 effectively suppressed the migration and invasion ability of endometrial cells, and the mechanism was also found out to be related to its function of inhibiting the autophagy of endometrial cells [68]. Based on the researches, we may conclude that autophagy play an important role in the migration and invasion of endometrial cells.

CA125 is a critical index which can reflect the active inflammatory responses and the development or stage, as well as predict the recurrence of endometriosis [97]. As is shown in the study of Ren et al. [98], Beclin 1 protein expression in eutopic endometrial tissues was negatively correlated with serum CA125 $(\mathrm{r}=-0.307, \mathrm{P}=0.015)$, and pelvic pain $(\mathrm{r}$ $=-0.542, \mathrm{P}=0.000)$. Lu et al. [38] also found that Beclin1 expression showed a negative correlation with the clinical stage of endometriosis, demonstrated that autophagy is lower in the advanced stage of endometriosis. However, our previous work showed that there was no significant difference in autophagy grade between stage I-II and stage III-IV endometriosis-derived ectopic ESCs [15]. Zheng et al. [24] clarified that there was a significant positive correlation between serum CA125 level and LC3-II protein level and/or LC3-II/LC3-I ratio in endometrial tissues, whereas the correlation index was separately 0.307 and 0.405 , and the CA125 level was negatively related to the mRNA expression of LC3B and Beclin and protein level of Beclin, while the correlation index was quite small and with no significance [24]. Since the relationship between autophagy and stage of endometriosis as well as the 
serum CA125 level remains to be controversial, more samples should be included in the research to figure out the fact, which may help to comprehend the role of autophagy and direct the future treatment of endometriosis with autophagy-related drugs.

\section{Hyperplasia}

Autophagy may be related to the endometrial hyperplasia induced by tamoxifen [99]. As a first generation of selective estrogen receptor modulators, tamoxifen is usually recommended for the hormone receptor positive breast cancer patients, but one of the side effects of tamoxifen is endometrial hyperplasia even endometrial cancer. Feng et al. found that tamoxifen could significantly induce the mRNA and protein expression of autophagy adaptor P62 in endometrial cells but not breast cancer cells, and inhibiting the expression of P62 retarded tamoxifenpromoted growth of endometrial cancer cells [99], indicating that the selectively promotion of autophagy in endometrial and breast cancer cells may be the reason for tamoxifen-induced hyperplasia. Actually, we may hypothesize that autophagy was associated with hyperplasia of endometrium, not only in the tamoxifen-induced cases. Studies are needed to prove whether this was true in the spontaneous hyperplasia or hormone-related hyperplasia since the evidence was rare.

\section{Endometrium-related infertility}

Although many factors contribute to infertility and early pregnancy loss, decidualization defects was regarded as an important factor contributing to compromised endometrial receptivity and poor implantation. As is discussed above, autophagy is beneficial to the process of decidualization, and impaired autophagy in endometrial cells may be related to infertility. In obese women, the increased level of palmitic acid may decrease the autophagy in endometrial cells as well as the process of decidualization, and further cause the implantation failure [16]. And autophagy in polycystic ovary syndrome patients was reported to be negative associated to the androgen level, indicating that the inspired autophagy may be related to the increased level of androgen, which inhibits the growth of follicle and is detrimental to the endometrial receptivity [34]. Much remains unknown in the field of infertility. Apart from the quality of zygote, the endometrium related process, including the decidualization, the implantation, the remodeling of uterus spiral artery and the endometrial receptivity, are all closely linked to a successful pregnancy. There's no doubt that the condition and function of endometrial or decidual stromal cells play a vital role, and autophagy activity of endometrial or decidual cells deserves more attention and may draw a new light on the way exploring the mechanisms of infertility.

\section{Conclusion and perspectives}

Autophagy is a conserved pathway in eukaryotic cells with the function to degrade misfolded proteins, aging organelles, and other damaged cell components, as well as participate in the regulation of biological activities such as proliferation, apoptosis, and immunoreaction. Endometrial cells undergo a dynamic autophagy in the periodic growth and shedding off during the menstrual cycle, and the autophagy level also changed in endometrium-related diseases, as well as the physiological processes including decidualization and atrophy. Although a lot has been done during the past years, much remains unknown related to the autophagy in endometrium. The researches about autophagy limit to the endometrium stromal cells and epithelial, while we believe that autophagy in endometrial immune cells was the same pivotal and even more important. What's more, autophagy influences different biological activities in the same disease or the same kind of cells, these effects may be mutually contradictory. The balance and relationship between these effects, or the synthetic role of autophagy at some certain conditions are still vague to our understanding. On the one side, autophagy could promote the drug resistance of tumor cells and the migration and invasion of ectopic endometrium; on the other side, autophagy may also induce the immune activation and cell apoptosis in the lesions area (Figure 1). There is no constant role of autophagy in different situation; at least the evidences were controversial related the function of autophagy even in the same diseases. For all this, there's no doubt that to determinate the law of autophagy is of great significance in improving the treatment and enhancing the medicine development in endometrium-related diseases.

\section{Acknowledgements}

This study was supported by the National Natural Science Foundation of China (NSFC) (No. 31671200, 91542108, 81471513 and 81471548), the Shanghai Rising-Star Program (No. 16QA1400800), the Oriented Project of Science and Technology Innovation from Key Lab. of Reproduction Regulation of NPFPC (CX2017-2), and the Program for Zhuoxue of Fudan University.

\section{Author Contributions}

Writing and editing of the manuscript and preparing the figures, S.L.Y.; editing the manuscript, 


\section{Normal menstrual cycle}

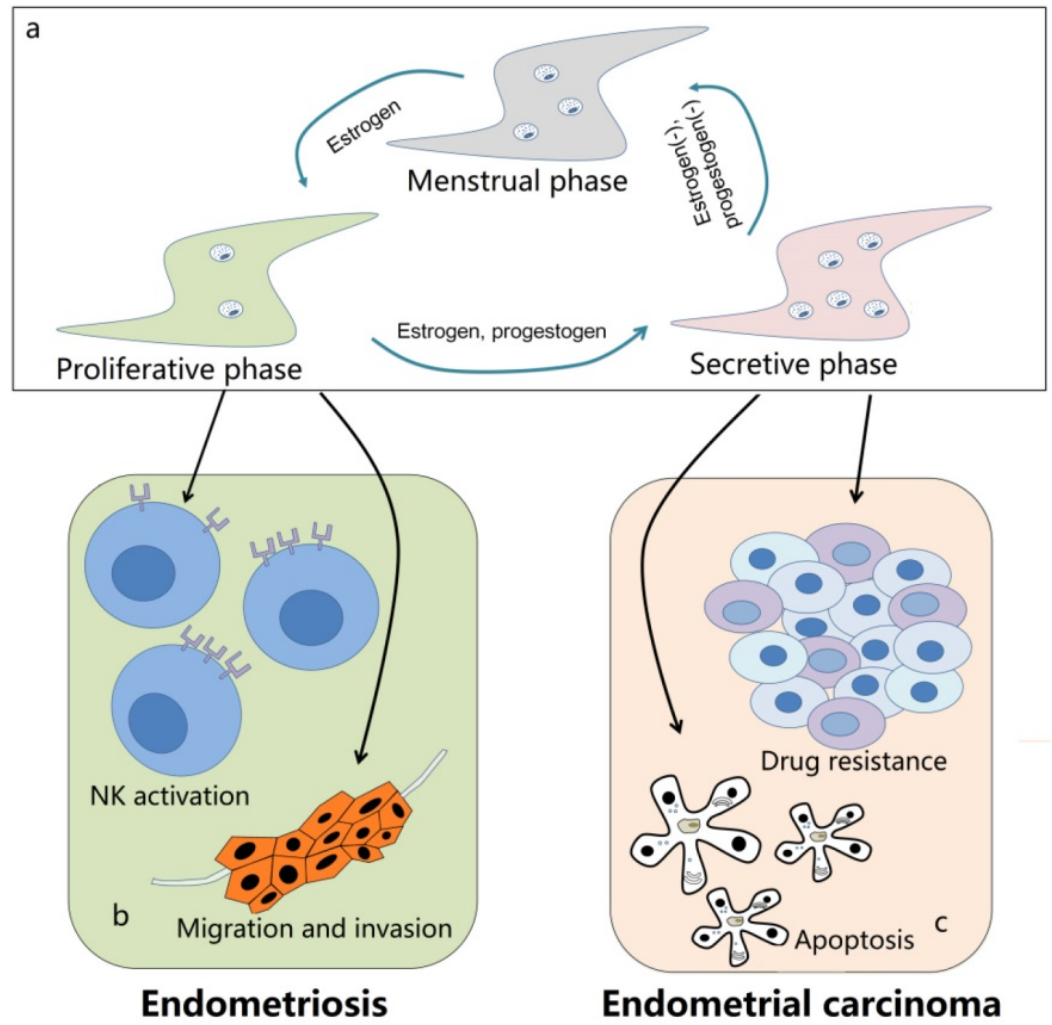

Figure 1. The role of autophagy in endometrium-related diseases. (a) The level of autophagy in normal menstrual cycle is in a dynamic state. In the proliferative phase, the autophagy activity is relatively low in endometrium cells. When entering into the secretive phase, it significantly increases to the peak in late secretive phase under the control of estrogen and progestogen. And during the menstrual phase, it remains a middle level with the withdrawal of estrogen and progestogen. (b) Autophagy is decreased in endometriosis. On the one hand, the downregulated autophagy can inhibit the activation of NK cells and induce the immune escape of ectopic endometrial stromal cells; on the other hand, the autophagy in endometrial cells may contribute to its migration and invasion. (c) In endometrial carcinoma, the upregulated autophagy is related to its resistance to several drugs, including sorafenib, cisplatin, paclitaxel and so on. Meanwhile, autophagy may also lead to apoptosis of tumor cells and is essential for some other treatment methods, such as metformin and suberoylanilide hydroxamic acid. Autophagy is a double-edged sword in endometrium-related diseases and need to be further explored.

H.Y.W. and D.J.L.; writing and editing of the manuscript and figures, M.Q.L.

\section{Competing Interests}

The authors have declared that no competing interest exists.

\section{References}

1. Gwangwa MV, Joubert AM, Visagie MH. Crosstalk between the Warburg effect, redox regulation and autophagy induction in tumourigenesis. Cellular \& Molecular Biology Letters. 2018;23(20):1-19

2. Zhang $\mathrm{Z}$, Lai Q, Li Y, et al. Acidic $\mathrm{pH}$ environment induces autophagy in osteoblasts. Scientific Reports. 2017;6(7):46161.

3. Zhong Z, Sanchez-Lopez E, Karin M. Autophagy, Inflammation, and Immunity: A Troika Governing Cancer and Its Treatment. Cell. 2016;166(2): 288-298.

4. Chun Y, Kim J. Autophagy: An Essential Degradation Program for Cellular Homeostasis and Life. Cells. 2018;7(12):1-26

5. Konrad L, Kortum J, Nabham R, et al. Composition of the Stroma in the Human Endometrium and Endometriosis. Reprod Sci. 2018;25(7): 1106-1115.

6. Amalinei C, Pavaleanu I, Grigoras A, et al. The endometrial regeneration frontiers: from mechanisms to applications in regenerative medicine. Rom J Morphol Embryol. 2018;59(2): 407-425

7. Valentijn AJ, Palial K, Al-Lamee H, et al. SSEA-1 isolates human endometrial basal glandular epithelial cells: phenotypic and functional characterization and implications in the pathogenesis of endometriosis. Hum Reprod. 2013;28(10): 2695-2708

8. Vanderstraeten A, Tuyaerts S, Amant F. The immune system in the normal endometrium and implications for endometrial cancer development. J Reprod Immunol. 2015;109: 7-16.

9. Zhou JZ, Way SS, Chen K. Immunology of the Uterine and Vaginal Mucosae. Trends Immunol. 2018;39(4): 302-314.
10. Choi J, Jo M, Lee E, et al. The role of autophagy in human endometrium. Biol Reprod. 2012;86(3): 70, 1-10

11. Ruiz A, Rockfield S, Taran N, et al. Effect of hydroxychloroquine and characterization of autophagy in a mouse model of endometriosis. Cell Death Dis. 2016;7: e2059.

12. Choi J, Jo M, Lee E, et al. Differential induction of autophagy by mTOR is associated with abnormal apoptosis in ovarian endometriotic cysts. Mol Hum Reprod. 2014;20(4): 309-317.

13. Zhou S, Zhao L, Yi T, et al. Menopause-induced uterine epithelium atrophy results from arachidonic acid/prostaglandin E2 axis inhibition-mediated autophagic cell death. Sci Rep. 2016;6: 31408

14. Choi $\mathrm{S}$, Shin $\mathrm{H}$, Song $\mathrm{H}$, et al. Suppression of autophagic activation in the mouse uterus by estrogen and progesterone. Journal of Endocrinology. 2014;221(1): 39-50.

15. Mei J, Zhu XY, Jin LP, et al. Estrogen promotes the survival of human secretory phase endometrial stromal cells via CXCL12/CXCR4 up-regulation-mediated autophagy inhibition. Hum Reprod. 2015;30(7): 1677-1689.

16. Rhee JS, Saben JL, Mayer AL, et al. Diet-induced obesity impairs endometrial stromal cell decidualization: a potential role for impaired autophagy. Hum Reprod. 2016;31(6): 1315-1326.

17. Mei J, Zhou WJ, Zhu XY, et al. Suppression of autophagy and HCK signaling promotes PTGS2(high) FCGR3(-) NK cell differentiation triggered by ectopic endometrial stromal cells. Autophagy. 2018;14(8): 1376-1397.

18. Zhang $\mathrm{L}$, Liu $\mathrm{Y}, \mathrm{Xu} \mathrm{Y}$, et al. The expression of the autophagy gene beclin-1 mRNA and protein in ectopic and eutopic endometrium of patients with endometriosis. Int J Fertil Steril. 2015;8(4): 429-436.

19. Sui X, Li Y, Sun $Y$, et al. Expression and significance of autophagy genes LC3, Beclin1 and MMP-2 in endometriosis. Exp Ther Med. 2018;16(3): 1958-1962.

20. Luo X, Cheng W, Wang S, et al. Autophagy Suppresses Invasiveness of Endometrial Cells through Reduction of Fascin-1. Biomed Res Int. 2018;2018: 8615435.

21. Allavena G, Carrarelli P, Del BB, et al. Autophagy is upregulated in ovarian endometriosis: a possible interplay with p53 and heme oxygenase-1. Fertil Steril. 2015;103(5): 1244-1251.e1241. 
22. Liu H, Du Y, Zhang Z, et al. Autophagy contributes to hypoxia-induced epithelial to mesenchymal transition of endometrial epithelial cells in endometriosis. Biol Reprod. 2018; 99(5): 968-981

23. Liu $\mathrm{H}$, Zhang $\mathrm{Z}$, Xiong $\mathrm{W}$, et al. Hypoxia-inducible factor-1alpha promotes endometrial stromal cells migration and invasion by upregulating autophagy in endometriosis. Reproduction. 2017;153(6): 809-820.

24. Zheng J, Luo X, Bao J, et al. Decreased Expression of HOXA10 May Activate the Autophagic Process in Ovarian Endometriosis. Reprod Sci. 2018: 1933719118768704

25. Borodkina AV, Shatrova AN, Deryabin PI, et al. Tetraploidization or autophagy: The ultimate fate of senescent human endometrial stem cells under ATM or p53 inhibition. Cell Cycle. 2016;15(1): 117-127.

26. Borodkina AV, Shatrova AN, Deryabin PI, et al. Calcium alterations signal either to senescence or to autophagy induction in stem cells upon oxidative stress. Aging (Albany NY). 2016;8(12): 3400-3418.

27. Du J, Zhu X, Guo R, et al. Autophagy induces G0/G1 arrest and apoptosis in menstrual blood-derived endometrial stem cells via GSK3-beta/beta-catenin pathway. Stem Cell Res Ther. 2018;9(1): 330, 1-13

28. Ran X, Zhou P, Zhang K. Autophagy plays an important role in stemness mediation and the novel dual function of EIG121 in both autophagy and stemness regulation of endometrial carcinoma JEC cells. Int J Oncol. 2017;51(2): 644-656.

29. Zhang B, Zhou WJ, Gu CJ, et al. The ginsenoside PPD exerts anti-endometriosis effects by suppressing estrogen receptor-mediated inhibition of endometrial stromal cell autophagy and NK cell cytotoxicity. Cell Death Dis. 2018;9(5): 574, 1-13

30. Gu CJ, Cheng J, Zhang B, et al. Protopanaxadiol and metformin synergistically inhibit estrogen-mediated proliferation and anti-autophagy effects in endometrial cancer cells. Am J Transl Res. 2017;9(9): 4071-4082.

31. Deng L, Feng J, Broaddus RR. The novel estrogen-induced gene EIG121 regulates autophagy and promotes cell survival under stress. Cell Death Dis. 2010;1: e32.

32. Choi J, Jo M, Lee E, et al. Dienogest enhances autophagy induction in endometriotic cells by impairing activation of AKT, ERK1/2, and mTOR. Fertil Steril. 2015;104(3): 655-664.e651.

33. Zhang D, Li J, Xu G, et al. Follicle-stimulating hormone promotes age-related endometrial atrophy through cross-talk with transforming growth factor beta signal transduction pathway. Aging Cell. 2015;14(2): 284-287.

34. Sumarac-Dumanovic M, Apostolovic M, Janjetovic K, et al. Downregulation of autophagy gene expression in endometria from women with polycystic ovary syndrome. Mol Cell Endocrinol. 2017;440: 116-124.

35. Chen Q, Gao R, Geng Y, et al. Decreased autophagy was implicated in the decreased apoptosis during decidualization in early pregnant mice. J Mol Histol. 2018;49(6): 589-597.

36. Yin B, Liu X, Guo SW. Caloric Restriction Dramatically Stalls Lesion Growth in Mice With Induced Endometriosis. Reprod Sci. 2018;25(7): 1024-1036.

37. Shi K, Wang $\mathrm{Q}, \mathrm{Su} \mathrm{Y}$, et al. Identification and functional analyses of differentially expressed metabolites in early stage endometrial carcinoma. Cancer Sci. 2018;109(4): 1032-1043.

38. $\mathrm{Lu} \mathrm{H}, \mathrm{Li} \mathrm{S}, \mathrm{Wu}$ Q. Retinoic acid regulates endometriotic stromal cell growth through upregulation of Beclin1. Arch Gynecol Obstet. 2018;297(1): 93-99.

39. Song $\mathrm{Y}, \mathrm{Du} \mathrm{Y}, \mathrm{Zou} \mathrm{W}$, et al. Involvement of impaired autophagy and mitophagy in Neuro-2a cell damage under hypoxic and/or high-glucose conditions. Sci Rep. 2018;8(1): 3301, 1-14

40. Wang X, Ribeiro M, Iracheta-Vellve A, et al. Macrophage-specific HIF-1alpha contributes to impaired autophagic flux in non-alcoholic steatohepatitis. Hepatology. 2019;0(0):1-19.

41. Bauckman KA, Haller E, Flores I, et al. Iron modulates cell survival in a Rasand MAPK-dependent manner in ovarian cells. Cell Death Dis. 2013;4: e592.

42. Ryter SW, Choi AM. Autophagy: An Integral Component of the Mammalian Stress Response. J Biochem Pharmacol Res. 2013;1(3): 176-188.

43. Filomeni G, De Zio D, Cecconi F. Oxidative stress and autophagy: the clash between damage and metabolic needs. Cell Death Differ. 2015;22(3): 377-388.

44. Talarico C, Dattilo V, D'Antona L, et al. SI113, a SGK1 inhibitor, potentiates the effects of radiotherapy, modulates the response to oxidative stress and induces cytotoxic autophagy in human glioblastoma multiforme cells. Oncotarget. 2016;7(13): 15868-84

45. Bai J-A, Xu G-F, Yan L-J, et al. SGK1 inhibits cellular apoptosis and promotes proliferation via the MEK/ERK/p53 pathway in colitis. World Journal of Gastroenterology. 2015;21(20): 6180-6193.

46. Conza D, Mirra P, Cali G, et al. The SGK1 inhibitor SI113 induces autophagy, apoptosis, and endoplasmic reticulum stress in endometrial cancer cells. Journal of Cellular Physiology. 2017;232(12): 3735-3743.

47. Lang F, Pelzl L, Hauser S, et al. To die or not to die SGK1-sensitive ORAI/STIM in cell survival. Cell Calcium. 2018;74: 29-34.

48. Conza D, Mirra P, Cali G, et al. The SGK1 inhibitor SI113 induces autophagy, apoptosis, and endoplasmic reticulum stress in endometrial cancer cells. J Cell Physiol. 2017;232(12): 3735-3743.

49. Kanda R, Hiraike H, Wada-Hiraike O, et al. Expression of the glucagon-like peptide-1 receptor and its role in regulating autophagy in endometrial cancer. BMC Cancer. 2018;18(1): 657, 1-11

50. Kao C, Chao A, Tsai CL, et al. Bortezomib enhances cancer cell death by blocking the autophagic flux through stimulating ERK phosphorylation. Cell Death Dis. 2014;5: e1510.
51. De U, Son JY, Sachan R, et al. A New Synthetic Histone Deacetylase Inhibitor, MHY2256, Induces Apoptosis and Autophagy Cell Death in Endometrial Cancer Cells via p53 Acetylation. Int J Mol Sci. 2018;19(9): 2743.

52. Liu $\mathrm{H}$, Zhang $\mathrm{L}$, Zhang $\mathrm{X}$, et al. PI3K/AKT/mTOR pathway promotes progestin resistance in endometrial cancer cells by inhibition of autophagy. Onco Targets Ther. 2017;10: 2865-2871.

53. Wang H, Li D, Li X, et al. Mammalian target of rapamycin inhibitor RAD001 sensitizes endometrial cancer cells to paclitaxel-induced apoptosis via the induction of autophagy. Oncol Lett. 2016;12(6): 5029-5035

54. Liu Y, Okamoto K. The TORC1 signaling pathway regulates respiration-induced mitophagy in yeast. Biochemical and Biophysical Research Communications. 2018;502(1): 76-83.

55. Manzoni C, Mamais A, Dihanich S, et al. mTOR independent alteration in ULK1 Ser758 phosphorylation following chronic LRRK2 kinase inhibition. Bioscience Reports. 2018;38: 1-12

56. Rahman MA, Terasawa M, Mostofa MG, et al. The TORC1-Nem1/Spo7-Pah1/lipin axis regulates microautophagy induction in budding yeast. Biochemical and Biophysical Research Communications. 2018;504(2): 505-512.

57. Tyler JK, Johnson JE. The role of autophagy in the regulation of yeast life span. Annals of the New York Academy of Sciences. 2018;1418(1): 31-43.

58. Zhou WJ, Chang KK, Wu K, et al. Rapamycin Synergizes with Cisplatin in Antiendometrial Cancer Activation by Improving IL-27-Stimulated Cytotoxicity of NK Cells. Neoplasia. 2018;20(1): 69-79.

59. Yan L, Zhou J, Gao Y, et al. Regulation of tumor cell migration and invasion by the H19/let-7 axis is antagonized by metformin-induced DNA methylation. Oncogene. 2015;34(23): 3076-3084

60. Tan BK, Adya R, Chen J, et al. Metformin Treatment Exerts Antiinvasive and Antimetastatic Effects in Human Endometrial Carcinoma Cells. The Journal of Clinical Endocrinology \& Metabolism. 2011;96(3): 808-816.

61. Zhuo Z, Wang A, Yu H. Metformin targeting autophagy overcomes progesterone resistance in endometrial carcinoma. Arch Gynecol Obstet. 2016;294(5): 1055-1061

62. Takahashi A, Kimura F, Yamanaka A, et al. Metformin impairs growth of endometrial cancer cells via cell cycle arrest and concomitant autophagy and apoptosis. Cancer Cell Int. 2014;14: 53, 1-12

63. Tsai HH, Lai HY, Chen YC, et al. Metformin promotes apoptosis in hepatocellular carcinoma through the CEBPD-induced autophagy pathway. Oncotarget. 2017;8(8): 13832-13845.

64. Li J, Gui Y, Ren J, et al. Metformin Protects Against Cisplatin-Induced Tubular Cell Apoptosis and Acute Kidney Injury via AMPKalpha-regulated Autophagy Induction. Sci Rep. 2016;6: 23975.

65. d'Abadie P, Borbath I, Goffette P, et al. Sorafenib Reduced Significantly Heptopulmonary Shunt in a Large Hepatocelullar Carcinoma. Clinical Nuclear Medicine. 2019;44(1): 70-71.

66. Wang H-T, Xia M. A meta-analysis of efficacy and safety of sorafenib versus other targeted agents for metastatic renal cell carcinoma. Medicine. 2019;98(1): e13779-e13779.

67. Eritja N, Chen BJ, Rodriguez-Barrueco R, et al. Autophagy orchestrates adaptive responses to targeted therapy in endometrial cancer. Autophagy. 2017;13(3): 608-624.

68. Li $Y$, Wang $X$, Wang $X$, et al. PDCD4 suppresses proliferation, migration and invasion of endometrial cells by inhibiting autophagy and NF-kappaB/MMP2/MMP9 signal pathway. Biol Reprod. 2018; 99(2):360-372

69. Lin $\mathrm{Q}$, Wang $\mathrm{Y}$, Chen $\mathrm{D}$, et al. Cisplatin regulates cell autophagy in endometrial cancer cells via the PI3K/AKT/mTOR signalling pathway. Oncol Lett. 2017;13(5): 3567-3571.

70. Tsubamoto $\mathrm{H}$, Inoue $\mathrm{K}$, Sakata $\mathrm{K}$, et al. Itraconazole Inhibits AKT/mTOR Signaling and Proliferation in Endometrial Cancer Cells. Anticancer Res. 2017;37(2): 515-519

71. Wu CH, Chen $\mathrm{HY}$, Wang $\mathrm{CW}$, et al. Isoliquiritigenin induces apoptosis and autophagy and inhibits endometrial cancer growth in mice. Oncotarget. 2016;7(45): 73432-73447.

72. Kang S, Kim JE, Song NR, et al. The ginsenoside 20-O-beta-D-glucopyranosyl-20(S)-protopanaxadiol induces autophagy and apoptosis in human melanoma via AMPK/JNK phosphorylation. PLoS One. 2014;9(8): e104305

73. Wang C, Liu B. miR-101-3p induces autophagy in endometrial carcinoma cells by targeting EZH2. Arch Gynecol Obstet. 2018;297(6): 1539-1548.

74. Martin-Mateos R, De Assuncao TM, Pablo Arab J, et al. Enhancer of Zeste Homologue 2 Inhibition Attenuates TGF-beta Dependent Hepatic Stellate Cell Activation and Liver Fibrosis. Cellular and Molecular Gastroenterology and Hepatology. 2019;7(1): 197-209.

75. Wei FZ, Cao Z, Wang X, et al. Epigenetic regulation of autophagy by the methyltransferase EZH2 through an MTOR-dependent pathway. Autophagy. 2015·11(12): 2309-2322

76. Xu TX, Zhao SZ, Dong M, et al. Hypoxia responsive miR-210 promotes cell survival and autophagy of endometriotic cells in hypoxia. Eur Rev Med Pharmacol Sci. 2016;20(3): 399-406.

77. Ran X, Yang J, Liu C, et al. MiR-218 inhibits HMGB1-mediated autophagy in endometrial carcinoma cells during chemotherapy. Int J Clin Exp Pathol. 2015;8(6): 6617-6626

78. Sun MY, Zhu JY, Zhang CY, et al. Autophagy regulated by lncRNA HOTAIR contributes to the cisplatin-induced resistance in endometrial cancer cells. Biotechnol Lett. 2017;39(10): 1477-1484. 
79. Liu H, Zhang Z, Xiong W, et al. Long non-coding RNA MALAT1 mediates hypoxia-induced pro-survival autophagy of endometrial stromal cells in endometriosis. J Cell Mol Med. 2019;23(1): 439-452.

80. Zeng $\mathrm{X}$, Yan $\mathrm{T}$, Schupp JE, et al. DNA mismatch repair initiates 6-thioguanine--induced autophagy through p53 activation in human tumor cells. Clin Cancer Res. 2007;13(4): 1315-1321.

81. $\mathrm{Xu} \mathrm{HL}, \mathrm{Xu} \mathrm{J}$, Zhang SS, et al. Temperature-sensitive heparin-modified poloxamer hydrogel with affinity to KGF facilitate the morphologic and functional recovery of the injured rat uterus. Drug Deliv. 2017;24(1): 867-881.

82. Yang D, Jiang T, Liu J, et al. CREB3 regulatory factor -mTOR-autophagy regulates goat endometrial function during early pregnancy. Biol Reprod. 2018;98(5): 713-721.

83. Yang D, Jiang T, Liu J, et al. Hormone regulates endometrial function via cooperation of endoplasmic reticulum stress and mTOR-autophagy. J Cell Physiol. 2018;233(9): 6644-6659.

84. Lebovitz CB, Robertson AG, Goya R, et al. Cross-cancer profiling of molecular alterations within the human autophagy interaction network. Autophagy. 2015;11(9): 1668-1687.

85. Sivridis E, Giatromanolaki A, Liberis V, et al. Autophagy in endometrial carcinomas and prognostic relevance of 'stone-like' structures (SLS): what is destined for the atypical endometrial hyperplasia? Autophagy. 2011;7(1): 74-82.

86. Hahne JC, Meyer SR, Dietl J, et al. The effect of Cordyceps extract and a mixture of Ganoderma lucidum/Agaricus Blazi Murill extract on human endometrial cancer cell lines in vitro. Int J Oncol. 2014;45(1): 373-382.

87. Fukuda T, Oda K, Wada-Hiraike $\mathrm{O}$, et al. The anti-malarial chloroquine suppresses proliferation and overcomes cisplatin resistance of endometrial cancer cells via autophagy inhibition. Gynecol Oncol. 2015;137(3): 538-545.

88. Liu S, Li X. Autophagy inhibition enhances sensitivity of endometrial carcinoma cells to paclitaxel. Int J Oncol. 2015;46(6): 2399-2408.

89. Singh AK, Bishayee A, Pandey AK. Targeting Histone Deacetylases with Natural and Synthetic Agents: An Emerging Anticancer Strategy. Nutrients. 2018;10(6): 731, 1-31

90. Orfanelli T, Jeong JM, Doulaveris G, et al. Involvement of autophagy in cervical, endometrial and ovarian cancer. Int J Cancer. 2014;135(3): 519-528.

91. Giatromanolaki A, Koukourakis MI, Koutsopoulos A, et al. High Beclin 1 expression defines a poor prognosis in endometrial adenocarcinomas. Gynecol Oncol. 2011;123(1): 147-151.

92. Zhan L, Li J, Wei B. Autophagy in endometriosis: Friend or foe? Biochem Biophys Res Commun. 2018;495(1): 60-63.

93. Yang HL, Mei J, Chang KK, et al. Autophagy in endometriosis. Am J Transl Res. 2017;9(11): 4707-4725.

94. Erekat NS. Autophagy precedes apoptosis among at risk cerebellar Purkinje cells in the shaker mutant rat: an ultrastructural study. Ultrastructural Pathology. 2018;42(2): 162-169.

95. Yu JJ, Sun HT, Zhang ZF, et al. IL15 promotes growth and invasion of endometrial stromal cells and inhibits killing activity of NK cells in endometriosis. Reproduction. 2016;152(2): 151-160.

96. Matsuzaki S, Pouly J-L, Canis M. In vitro and in vivo effects of MK2206 and chloroquine combination therapy on endometriosis: autophagy may be required for regrowth of endometriosis. British Journal of Pharmacology. 2018;175(10): 1637-1653.

97. Mihalyi A, Gevaert O, Kyama CM, et al. Non-invasive diagnosis of endometriosis based on a combined analysis of six plasma biomarkers. Hum Reprod. 2010;25(3): 654-664.

98. Ren Y, Mu L, Ding X, et al. Decreased expression of Beclin 1 in eutopic endometrium of women with adenomyosis. Arch Gynecol Obstet. 2010;282(4): 401-406.

99. Feng L, Li J, Yang L, et al. Tamoxifen activates Nrf2-dependent SQSTM1 transcription to promote endometrial hyperplasia. Theranostics. 2017;7(7): 1890-1900. 